\title{
Galactus - Um ambiente inteligente para apoio à tomada de decisão no âmbito do Ministério Público de Sergipe
}

\author{
Fabio Mangueira da Cruz Nunes ${ }^{1}$, Methanias Colaço Júnior ${ }^{1}$, José Bonifacio da \\ Silva Junior ${ }^{1}$, Luan Bruno B. S. Costa ${ }^{1}$, Everton Carlos S. Recchi ${ }^{2}$ \\ ${ }^{1}$ Departamento de Sistemas de Informação \\ Universidade Federal de Sergipe (UFS). Itabaiana- SE - Brasil \\ ${ }^{2}$ Departamento de Ciência da Computação \\ Universidade Tiradentes (UNIT). Aracaju - SE - Brasil. \\ fabio.dipolcgi@gmail.com,mjrse@ hotmail.com, josebonifacio@mpse.mp.br, \\ luanbrunos@gmail.com, everton.recchi@hotmail.com
}

\begin{abstract}
Galactus is a designed, integrating environment with the capacity to store all the digital collection of investigative bodies or departments that operate with the activity of Public Security Intelligence (PSI). The Prosecution Office of Sergipe has used this technological environment to prospect and discover essential information to the investigative process. Daily case studies have presented results that demonstrate the inherent need of the investigator to possess a unique modal of search.
\end{abstract}

Resumo. O Galactus é um ambiente projetado, integrador, com capacidade para armazenar todo o acervo digital investigativo de órgãos ou departamentos que atuam com a atividade de Inteligência de Segurança Pública (ISP). O Ministério Público de Sergipe tem utilizado este ambiente tecnológico para prospecção e descoberta de informações essenciais ao processo investigativo. Estudos de casos diários têm apresentado resultados que evidenciam a necessidade inerente ao investigador de possuir um único modal customizável de busca.

\section{Introdução}

O termo Data Warehouse (DW), ou Armazém de Dados, é utilizado para nomear um "banco de dados histórico, separado do ambiente de produção da organização e projetado para apoio à decisão" (Colaço Jr., 2004). As experiências acadêmica e de mercado dos últimos vinte anos têm apontado fatores primordiais para o sucesso de um projeto de DW (Colaço et al., 2009) (Kimball, 2011) (Costa et al., 2015) (Costa et al, 2016).

No atual contexto Big Data, o DW perdeu o protagonismo e passou a ser o penúltimo passo do processo de descoberta de conhecimento, servindo como um ambiente que viabiliza a eficiência das análises baseadas em hipóteses oriundas do grande volume de dados explorado previamente. Estas evolução e mutação são destacadas a seguir.

\subsection{Big Data e Data Analytics}

O termo Data Analytics ganhou força nos últimos anos devido à democratização da conectividade e a consequente explosão de informações armazenadas, gerando o fenômeno conhecido como Big Data (Mcafee et al., 2012). Isto implica necessidade de profissionais de inteligência com conhecimentos de matemática, mineração de dados, amostragem e estatística inferencial, pois será inviável analisar todos os dados disponíveis 
(Lalanne, 2016). Neste contexto, enquanto técnicas de Data Mining identificam padrões e informações ocultas que auxiliam a consecução procedimental no âmbito investigativo, em paralelo, técnicas de Data Analytics podem se concentrar apenas na seleção das amostras verídicas e valorosas do Big Data, bem como nas inferências, derivando conclusões baseadas no que já foi descoberto pelo investigador.

Desta forma, independente do volume de dados, as soluções de inteligência continuam sendo beneficiadas quando da existência de um banco de dados histórico e separado do ambiente de produção (em outras palavras, um DW). Todavia, ter este banco histórico não é garantia de que ele foi bem projetado, está sendo usado e, se estiver, se está alinhado com a estratégia da organização.

Para Silva, Silva \& Gome (2016), a dificuldade de gerir dados e informações que sejam analisadas e interpretadas de forma adequada e eficaz, objetivando auxiliar um processo de tomada de decisão, é comum nos ambientes organizacionais. Muitas empresas obtêm diversos dados sobre o seu negócio e mercado que está inserido, no entanto, não conseguem transformá-los em informações relevantes e estratégicas para melhores decisões. O objetivo do ambiente apresentado na próxima seção é o de mitigar estes problemas de gestão de dados integrados.

\section{O Galactus}

A infraestrutura do Galactus foi desenvolvida em um ambiente coorporativo de governo, o Ministério Público de Sergipe, com a premissa de integrar, de forma amigável, diversas fontes de dados capazes de trazer aos investigadores, promotores de justiça e demais usuários, agilidade na obtenção das informações.

A base do ambiente é um sistema informacional pautado em web application, composto por containers de dados formadores de um DW (Colaço et al., 2009) que encapsula informações cadastrais e de diversas investigações.

Essa integração permite o vínculo automático e interativo de procedimentos investigativos, tais como, por exemplo, os procedimentos criminais. Os benefícios perpassam a celeridade de atuação e persecução processual penal, provendo à atividade investigativa uma ferramenta de atuação operacional capaz de descobrir conhecimento e gerar novas hipóteses investigativas.

\section{Funcionamento do Galactus}

O Galactus foi desenvolvido na linguagem de programação PHP (Hypertext Preprocessor), utilizando como Framework o Laravel, que por sua vez utiliza como padrão de arquitetura o MVC (Model-View-Controller). Além disto, o front-end conta com módulos funcionais específicos, são eles:

- Admin: responsável por páginas de administração, gerência de usuários e controle de perfis e permissões;

- Auditoria: módulo responsável por toda parte de auditoria das pesquisas que são realizadas pelos usuários;

- Busca Empresa: responsável por toda lógica de pesquisa das pessoas jurídicas;

- Busca Pessoa: responsável pela lógica das pesquisas de pessoas físicas;

- Dashboard: módulo que lida com a página após a autenticação do usuário, trazendo informações sobre as pesquisas que este realiza (vide Figura 1); 


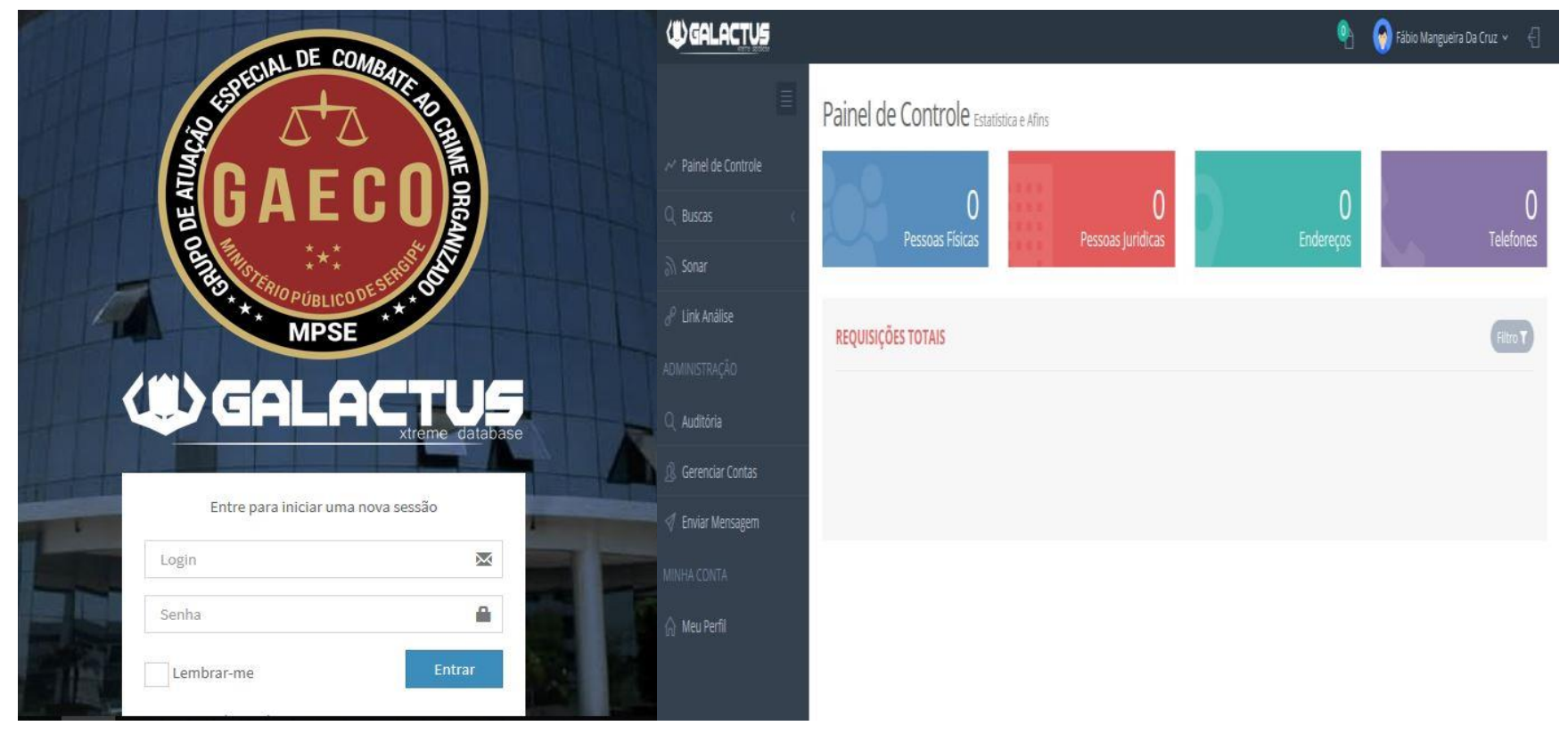

Figura 1. Tela de Login e Painel Inicial Galactus.

- Link-Análise: responsável pela página de análise de vínculos (vide Figura 2);

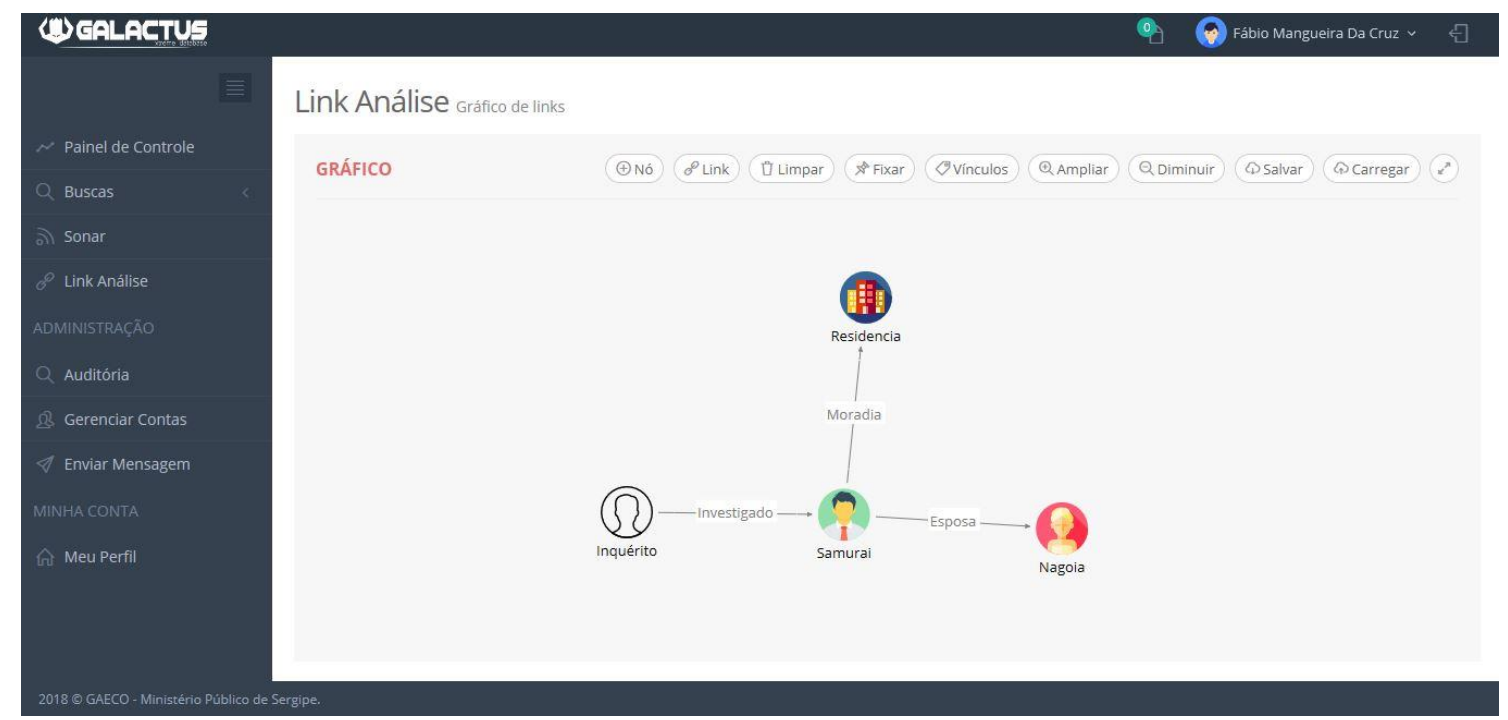

Figura 2. Link-Análise

- Orcrim: responsável pela lógica de pesquisas sobre as organizações criminosas;

- Relatório: módulo responsável pela administração e geração de relatórios do sistema;

- Requisição: módulo que serve como base para as requisições ao GalactusCore dos outros módulos;

- Sonar: módulo responsável pela parte de monitoramento de um investigado;

- User: módulo que se responsabiliza pelos usuários do sistema. 
Quanto ao Background, o GalactusCore, desenvolvido na linguagem Java, compreende três módulos principais: provedores, serviços Web e motores de busca. Os provedores são responsáveis pelo processo de ETL (Extract, Transform and Load), o que significa que a extração realizada parte de fontes de dados variadas, incluindo redes sociais e web em geral. Em relação aos serviços web, estes (webservices) executam as requisições, que por sua vez usam os motores de busca para processamento dos dados.

\section{Conclusão}

A ferramenta Galactus tem sido relevante para o desenvolvimento de atividades investigativas neste novo contexto social. Usuários especialistas entrevistados reforçaram o quão é importante ter menor custo para acessar as diversas bases que descrevem todo o ciclo de vida de uma empresa ou pessoa investigada, considerando as suas conexões.

Ademais, é importante ressaltar que a evolução desta ferramenta robustece a necessidade da manutenção de uma base histórica, que, num futuro próximo, proporcionará a exploração do acoplamento de novas técnicas de Inteligência Artificial e Mineração de Dados.

Em suma, os trabalhos futuros englobarão uma melhor modelagem para o módulo de Link-Análise, a qual garanta uma visualização exitosa para dados em grandes escalas, bem como automação de consultas de mineração de texto e de dados que atuem na busca de padrões textuais fraudulentos e indícios de Lavagem de Dinheiro.

\section{Referências}

COLAÇO JÚNIOR, M. Projetando Sistemas de Apoio à Decisão Baseados em Data Warehouse. Rio de Janeiro: Axcel Books do Brasil Editora, 2004.

COLAÇO Jr., Methanias; MENDONÇA, M. G.; RODRIGUES, F. Data Warehousing in an Industrial Software Development Environment. In: IEEE/NASA Software Engineering Workshop, 2009, Skövde.

COSTA, J. K. G., SANTOS, I. P. O., COLAÇO Jr., M.. and NASCIMENTO, A. V. R. P.. Experimentação na Indústria para Aumento da Efetividade da Construção de Procedimentos ETL em um Ambiente de Business Intelligence. SBSI - Simpósio Brasileiro de Sistemas de Informação, 2015.

COSTA, J. K. G., SANTOS, I. P. O., COLAÇO Jr., M.. and NASCIMENTO, A. V. R. $P$.. Um experimento em um ambiente de Business Intelligence industrial para melhoria da manutenção de cargas de dados. SBSI - Simpósio Brasileiro de Sistemas de Informação, 2016.

KIMBALL, R. and M. Ross. The data warehouse toolkit: the complete guide to dimensional modeling. John Wiley \& Sons, 2011.

LALANNE, Christophe. Statistical Data Analytics. Foundations for Data Mining, Informatics, and Knowledge Discovery, Journal of Statistical Software, 069, 2016.

MCAFEE, A. E. Brynjolfsson, T. H. Davenport, D. Patil, and D. Barton. Big data. The management revolution. Harvard Bus Rev, 90(10):61-67, 2012.

SILVA, R. A. d.; SILVA, F. C. A.; GOME, C. F. S. O uso do business intelligence (bi) em sistema de apoio à tomada de decisão estratégica. GEINTEC, 2016. 